POS $\quad$ PROCEEDINGS

\title{
An undiscovered pulsar in the Local Bubble as an explanation of the local high energy cosmic ray all-electron spectrum
}

Rubén López-Coto*

INFN Padova

E-mail: rlopezepd.infn.it

Dan Parsons, Jim Hinton, Gwenael Giacinti

Max Planck Institute for Nuclear Physics, Heidelberg, Germany

36th International Cosmic Ray Conference -ICRC2019-

July 24th - August 1st, 2019

Madison, WI, U.S.A.

${ }^{*}$ Speaker. 
Cosmic ray electrons and positrons are tracers of particle propagation in the interstellar medium (ISM). A recent measurement performed using H.E.S.S. extends the all-electron (electron+positron) spectrum up to $20 \mathrm{TeV}$, probing very local sources and transport due to the $10 \mathrm{kyr}$ cooling time of these particles. An additional key local measurement was the recent estimation of the ISM diffusion coefficient around Geminga performed using HAWC. The inferred diffusion coefficient is much lower than typically assumed values. It has been argued that if this diffusion coefficient is representative of the local ISM, pulsars would not be able to account for the all-electron spectrum measured at the Earth. Here we show that a low diffusion coefficient in the local ISM is compatible with a pulsar wind nebula origin of the highest energy electrons, if a so far undiscovered pulsar with spin-down power $\sim 10^{34} \mathrm{erg} / \mathrm{s}$ exists within 30 to $80 \mathrm{pc}$ of the Earth. The existence of such a pulsar is broadly consistent with the known population and may be detected in near future survey observations. The results included in this proceeding and shown in this presentation were published in [1]. The details about this analysis can be found in the aforementioned reference.

\section{References}

[1] López-Coto, R., et al., Undiscovered pulsar in the Local Bubble as the explanation of the local all-electron spectrum, Phys. Rev. Lett., 121, 251106. 